\title{
An evaluation of advance care planning during the COVID-19 pandemic: a retrospective review of patient involvement in decision making using routinely collected data from digital ReSPECT records
}

\author{
Authors: Adam Hurlow, ${ }^{\mathrm{A}}$ Lucy Wyld ${ }^{\mathrm{A}}$ and Andrew Breen ${ }^{\mathrm{B}}$
}

\section{Objective}

To review advance care planning (ACP) practice during the COVID-19 pandemic, evaluating the number of plans created, patient participation, cardiopulmonary resuscitation recommendations and variation between different population groups.

Design

A retrospective analysis and comparison of routinely collected data from electronic recommended summary plan for emergency care and treatment (ReSPECT) records documented in April 2020 and January to December 2019.

\section{Setting/participants}

Electronic ReSPECT documents completed for adult patients at a large, acute hospital trust in the UK.

\section{Results}

The number of plans created per 1,000 admissions in April 2020 was $333.0 \%$ higher than in 2019. A greater proportion of plans created during April 2020 were discussed with the patient and the proportion containing a 'for cardiopulmonary resuscitation' recommendation was higher across all population groups. A greater proportion of plans were created for younger adults and Black and minority ethnic groups during the pandemic.

\section{Conclusion}

Increased ACP during a crisis can be achieved alongside increased patient participation in decision making. A tool such as ReSPECT that supports recommendations for, as well as limitations on, treatment may have enabled the expansion of ACP observed.

KEYWORDS: advance care planning, patient participation, cardiopulmonary resuscitation, COVID-19, decision making

DOI: $10.7861 /$ clinmed.2020-1036

Authors: ${ }^{A}$ consultant in palliative medicine, Leeds Teaching Hospitals NHS Trust, Leeds, UK; ${ }^{B}$ consultant in intensive care medicine and anaesthesia, Leeds Teaching Hospitals NHS Trust, Leeds, UK

\section{Introduction}

People with serious illnesses are at risk of worse outcomes following COVID-19 infection and may not benefit from all possible treatments. Advance care planning (ACP), the agreement of treatment recommendations ahead of clinical deterioration, is therefore a key part of the pandemic response., ${ }^{1,2}$ This process involves a holistic person-centred approach in which individualised care preferences and treatment recommendations are discussed ahead of potential clinical deterioration. ACP increases the likelihood that interventions are concordant with individual preferences and of clinical benefit. 3.4 When facilitated with compassion, adopting an individualised approach, ACP empowers patients and improves experience of care. ${ }^{5}$ However, concern has been expressed about 'blanket' treatment limitation, such as 'do not attempt cardiopulmonary resuscitation' (DNACPR) recommendations, that target specific and have been made without consultation or ignore the individual's circumstances, views and preferences. ${ }^{6}$

To evaluate ACP at the onset of the pandemic, as part of quality assurance and improvement processes, investigators in a large acute hospital trust retrospectively analysed routinely collected data from recommended summary plan for emergency care and treatment (ReSPECT) plans for adult patients ( 16 years and over). ReSPECT is an ACP initiative in the UK that includes a standardised form for the documentation of treatment recommendations irrespective of diagnosis and prognosis.? ReSPECT was implemented digitally within the trust's electronic record in 2018. We compared ReSPECT forms completed or initiated during the month of April 2020 with the previous year (1 January to 31 December 2019).

At the onset of the pandemic, in the setting of a potentially fatal illness, clinicians were encouraged at both national and local level to consider timely, patient-centred discussions addressing advance care planning. This was of particular importance in patients at risk of significant clinical deterioration, including the elderly and those living with severe frailty or underlying health conditions such as hypertension, diabetes, cardiovascular disease or chronic lung disease. The outcomes of ACP discussions were documented in ReSPECT plans within patients' electronic health records. 


\section{Methods}

Data were collected and analysed on 18 June 2020 using a Microsoft Analysis Services reporting tool that collates selected data from both digital ReSPECT plans and the hospital patient administrative system. Only plans initiated or amended during the specified periods were analysed. Those created before the periods, but not amended during them, were excluded.

The following variables were extracted: age ( $>65$ and $<65$ years old); ethnicity (as per Office of National Statistics principles); sex; setting (inpatient/outpatient); patient participation; and cardiopulmonary resuscitation (CPR) recommendations. ${ }^{8,9}$ Due to the rapidity of the review, and quality improvement focus, investigators did not directly assess plans, analyse raw data or include additional data items not extracted by the reporting tool.

Rarely, a patient had two current iterations of ReSPECT, either because they had a pilot-phase plan and a plan created after the pilot, or because they had dual records that had been consolidated. As there were more ReSPECT plans than patients, patient characteristics are presented as a proportion of the total number of patients with ReSPECT while information from ReSPECT is presented as a proportion of the total number of plans.

\section{Results}

\section{Number of ReSPECT plans and patients}

In April 2020, 1,636 patients had ReSPECT initiated or amended, generating 1,637 plans, compared to 7,868 patients and 7,875 plans, 655.7 patients/month, at baseline (Fig 1). The majority were new plans initiated in the respective periods; 1,499/1,637 (91.6\%) in April 2020 and 7,670/7,875 (97.4\%) in 2019.

The prevalence of ACP among hospital inpatients increased by 333.0\% during the pandemic: in April 2020, 4,009 adults were admitted and 1,118 inpatient plans were made, amounting to 278.9 ReSPECT plans per 1,000 inpatients compared 84.0 plans per 1,000 patients $(7,875 / 93,751)$ at baseline.

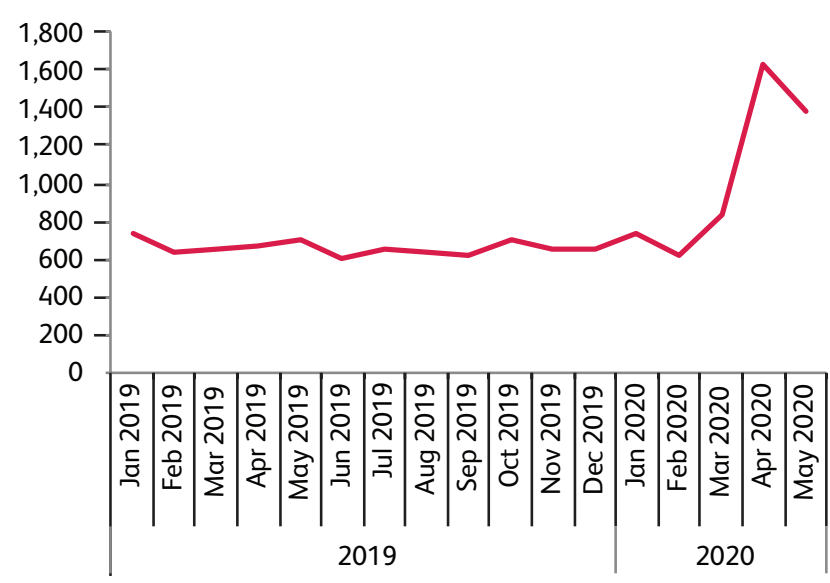

Fig 1. Total number of patients with ReSPECT plans initiated/amended per month, demonstrating an increase in the number of patients with ReSPECT plans initiated/amended during the first surge in the COVID-19 pandemic. In April 2020, 1,636 patients had ReSPECT initiated or amended, generating 1,637 plans, compared to a total of 7,868 patients and 7,875 plans during $2019,655.7$ patients per month at baseline.

\begin{tabular}{|c|c|c|c|}
\hline Ethnicity & Number & Leeds (\%) & England (\%) \\
\hline White & 639,487 & 85 & 85.5 \\
\hline Asian & 58,243 & 7.7 & 7.7 \\
\hline Black & 25,893 & 3.5 & 3.4 \\
\hline Mixed & 19,632 & 2.7 & 2.2 \\
\hline Other & 8,230 & 1.1 & 1 \\
\hline Unknown & 0 & 0 & 0.2 \\
\hline
\end{tabular}

\section{Demographics}

In April 2020, 1,052 (64.3\%) patients were over 65 years old compared to 6,788 (86.3\%) in 2019. In April 2020, 793 (48.5\%) were female compared to 4,357 (55.4\%) in 2019. The proportions of people from different ethnic groups in April 2020 and 2019 overall were:

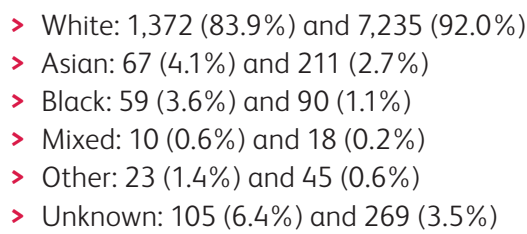

The ethnic make up of the population the trust serves is comparable to National data (Table 1$).{ }^{10}$

\section{Patient participation in ReSPECT}

In April 2020, 1,196 (73.1\%) of ReSPECT plans were made with the patient, compared to 4,558 (57.9\%) in 2019. During the pandemic, $426(26.0 \%)$ plans were made in the best interests of patients who lacked capacity compared to 3,224 (42\%) in 2019.

In April 2020, 575 (72.5\%) of women's plans were made with their participation compared to $621(73.6 \%)$ of men's plans. In $2019,2,521(58.0 \%)$ and 2,032 (58.0\%) of plans were made with female and male patients respectively. $497(85.1 \%)$ of plans for those $16-65$ years of age were made with their participation compared to $646(59.7 \%)$ in 2019. Though the proportion made with, rather than for, patients over 65 in April 2020, 699 (64.5\%), was lower than those under 65 , this was an increase on 2019 when only $3,912(57.6 \%)$ plans for those over 65 years were made with patient participation.

During the pandemic, $1,000(72.8 \%)$ plans for White ethnic populations were made with their participation. The proportions of plans made on this basis for other populations in April 2020 and 2019 were:

> Mixed: 10 (100\%) and $12(66.7 \%)$

> Asian: $49(73.1 \%)$ and $83(39.3 \%)$

> Black: $40(67.8 \%)$ and $38(42.2 \%)$

> Other: $20(87.0 \%)$ and $12(66.7 \%)$

> Unknown: 77 (73.4\%) and 125 (45.6\%)

\section{CPR recommendation}

In April 2020, 1,039 (63.5\%) plans contained a DNACPR recommendation compared to $7,670(97.4 \%)$ in 2019 . The 
remaining CPR recommendations in April 2020 and 2019 overall were:

> For attempted CPR: 591 (36.1\%) and $116(1.5 \%)$

$>$ No formal recommendation: $1(0.1 \%)$ and $28(0.4 \%)$

> Not completed: 6 (0.4\%) and $61(0.8 \%)$

In April 2020, a greater proportion of women's plans recommended DNACPR, 534 (67.3\%), compared to 505 (59.8\%) of men's plans. In 2019 4,255 (97.6\%) and 3,404 (97.2\%) of plans for women and men respectively recommended DNACPR.

In April 2020, 149 (25.5\%) plans for those aged $16-65$ years recommended DNACPR compared to 701 (84.5\%) plans for those aged over 65 years. In 2019 this was 1,029 (95.0\%) plans for those aged $16-65$ years and 6,641 (97.8\%) plans for those aged 65 years or over.

922 (67.2\%) plans for the White ethnic group specified DNACPR in April 2020 and 7,060 (97.5\%) in 2019 compared to the following proportions for other groups:

> Asian: 30 (44.8\%) and $199(94.3 \%)$

$>$ Black: $11(40 \%)$ and $86(95.6 \%)$

> Mixed: 2 (20.0\%) and $17(94.4 \%)$

> Other: $10(43.5 \%) 42(93.35)$

> Unknown: 52 (49.5\%) and $266(97.4 \%)$

In April 2020, fewer plans, 644 (53.8\%), made with the participation of the patient recommended DNACPR than those made for patients without capacity, 385 (90.4\%). The proportion of plans for those without capacity recommending DNACPR had fallen from 2019 baseline of 3,161 (98.1\%)

Those plans made for patients without capacity recommending DNACPR were discussed as follows in April 2020 and 2019 overall:

> Discussed with relative and/or carer: 292 (75.8\%) April 2020 and 2,806 (88.7\%) 2019

$>$ Emergency decision and unable to contact patient representative: 75 (19.5\%) and $266(8.4 \%)$

> Discussed with patient: 18 (4.7\%) and $89(2.8 \%)$

\section{Conclusion}

In this single centre, the proportion of patients who received ACP increased during the pandemic. A greater proportion of ReSPECT plans were made with the patient during this period when compared to baseline. This increase was seen across all demographic groups analysed. The proportion of patients who participated in ACP during the pandemic increased from baseline notably more for patients aged 65 years and under.

The patient group who received ACP during the pandemic were younger and more ethnically diverse than at baseline. This may reflect changing characteristics of people accessing hospital care due to reduced non-COVID-19 activity and the differential impact of COVID-19 on people from Black, Asian and minority ethnic populations. ${ }^{9,11}$ As ReSPECT supports recommendations for interventions, as well as advising limits on treatment, and can be created for anyone irrespective of prognosis and diagnosis, its use may have enabled the expansion of advance care planning seen in response to the pandemic.

The proportion of plans containing a DNACPR recommendation was lower in all demographic groups during the pandemic. This may be partially explained by the reduced proportion of patients over 65 years who received ACP, as advancing age is associated with worse CPR outcomes and more DNACPR recommendations. ${ }^{12,13}$

During the pandemic, those aged over 65 had a notably higher proportion of DNACPR recommendations, Black, Asian and minority ethnic groups had a lower proportion compared to the majority White population and women had a higher proportion than men. While differences based on age, gender and ethnicity have been observed in other studies the discrepancy between men and women, and younger and older groups widened during the pandemic. 14,15

The proportion of plans containing a DNACPR recommendation fell irrespective of the level of patient participation. However, the fall was greatest among plans made with patients compared to those made for patients without capacity. While lack of capacity correlates with older age and multimorbidity, both associated with worse CPR outcomes, this in itself does not explain the increasing discrepancy in pandemic period. ${ }^{16}$ The proportion of plans for patients without capacity specifying DNACPR that were made on an emergency basis was higher during the pandemic than baseline. This may reflect the high risk of rapid deterioration and challenges contacting patient representatives due to restricted visiting.

This study evaluates ACP practice across a large NHS organisation. The findings may not reflect other settings and therefore further detailed evaluation across care settings, capturing broader demographic and clinical information alongside patient experience, is necessary to understand the impact of the pandemic on practice.

\section{References}

1 Zhou F, Yu T, Du R et al. Clinical course and risk factors for mortality of adult inpatients with COVID-19 in Wuhan, China: a retrospective cohort study. Lancet 2020;395:1054-62.

2 Curtis JR, Kross EK, Stapleton RD. The importance of addressing advance care planning and decisions about do-not-resuscitate orders during novel coronavirus 2019 (COVID-19). JAMA 2020; 323:1771-2

3 Zwakman M, Jabbarian LJ, van Delden J] et al. Advance care planning: a systematic review about experiences of patients with a life-threatening or life-limiting illness. Palliat Med 2018; 32:130521.

4 Lightbody C], Campbell JN, Herbison GP et al. Impact of a treatment escalation/limitation plan on non-beneficial interventions and harms in patients during their last admission before in-hospital death, using the Structured Judgment Review Method. BMJ Open 2018:8:e024264.

5 Jimenez G, Tan WS, Virk AK et al. Overview of systematic reviews of advance care planning: summary of evidence and global lessons. J Pain Symptom Manage 2018:56:436-59.

6 Bledsoe TA, Jokela JA, Deep NN et al. Universal do-not-resuscitate orders, social worth, and life-years: opposing discriminatory approaches to the allocation of resources during the COVID-19 pandemic and other health system catastrophes. Ann Intern Med 2020;173:230-2.

7 Fritz Z, Pitcher D, Regnard C, Spiller J, Wang M. ReSPECT is a personal emergency care plan summary. BMJ 2017;357:j2213.

8 Office for National Statistics. Harmonised concepts and questions for social data sources. Primary principles. ONS, 2015.

9 Propper C, Stoye G, Zaranko B. The wider impacts of the coronavirus pandemic on the NHS. Fisc Stud 2020, in press (DOI: 10.1111/1475-5890.12227).

10 Leeds Observatory. Population of Leeds. https://observatory.leeds. gov.uk/population/ (Accessed 19 February 2021).

11 Lassale C, Gaye B, Hamer M et al. Ethnic disparities in hospitalisation for COVID-19 in England: the role of socioeconomic 
factors, mental health, and inflammatory and pro-inflammatory factors in a community-based cohort study. Brain Behav Immun 2020;88:44-9.

12 Chevaux F, Gagliano M, Waeber G et al. Patients' characteristics associated with the decision of 'do not attempt cardiopulmonary resuscitation' order in a Swiss hospital. Eur ] Intern Med 2015;26:311-6.

13 Fernando SM, Tran A, Cheng W et al. Pre-arrest and intra-arrest prognostic factors associated with survival after in-hospital cardiac arrest: systematic review and meta-analysis. BMJ 2019;367:16373.

14 Perman SM, Beaty BL, Daugherty SL et al. Do sex differences exist in the establishment of 'do not attempt resuscitation' orders and survival in patients successfully resuscitated from in-hospital cardiac arrest. J Am Heart Assoc 2020; 9:e014200.
15 Rahemi Z, Williams CL. Does ethnicity matter - Cultural factors underlying older adults' end-of-life care preferences: A systematic review. Geriatr Nurs 2020:41:89-97.

16 Murphy R, Fleming S, Curley A et al. Who can decide? Prevalence of mental incapacity for treatment decisions in medical and surgical hospital inpatients in Ireland. QJM 2018;111:881-5.

Address for correspondence: Dr Adam Hurlow, Palliative Care Team, Floor 1 Robert Ogden Centre, St James's University Hospital, Leeds, West Yorks, LS9 7TF, UK.

Email: a.hurlow@nhs.net 